\title{
Noise in HgCdTe LWIR arrays
}

\author{
F.F. Sizov, A.G. Golenkov, V.V. Zabudsky, V.P. Reva \\ Institute of Semiconductor Physics, 41 prospect Nauky, 03028 Kiev, Ukraine; e-mail: sizov@isp.kiev.ua
}

\begin{abstract}
Mercury cadmium telluride (MCT) hybrid arrays for long-wavelength infrared (LWIR) applications with $n^{+}$-p-diodes and $n$-channel charged coupled devices (CCD) silicon readouts were designed, manufactured and tested. Performance of these arrays at $T \approx 80 \mathrm{~K}$ is considered.

The measurements of noise and signal-to-noise ratio (SNR) are the key issues to determine performance parameters to characterize IR-sensors. That puts certain requirements to the registration system and used methods of measuring signals. To find out and eliminate noise sources the spectral noise power of signals was analyzed. It allowed the possibility to implement actions for reducing of the registration system noise, and to define the software noise filters to be used.
\end{abstract}

Keywords: MCT multielement linear arrays, noise measurements, detectivity, CCD readouts.

Paper received 02.08.02; accepted for publications 17.12.02.

\section{Introduction}

Today high performance infrared (IR) imaging systems typically include hybrid focal plane array (FPAs) cooled down to cryogenic temperatures with a signal processors (read out devices) in the focal plane. In spite of the complications involved in the physics of MCT material (intensive Shockley-Read-Hall recombination and trap-assisted tunneling due to a large number of different types of defects in the gap) $\mathrm{HgCdTe}$ moderately cooled arrays remain the most sensitive small pitch FPAs among others (e.g., QWIP (both AlGaAs QWIPs and GaInSb strain layer superlattices), doped silicon detectors, uncooled microbolometer arrays, InSb photodiodes, Schottky-barrier detectors, etc.) in LWIR and MWIR regions operating at high frame rates and low integration time. Now $\mathrm{HgCdTe}$ solid solution is nearly ideal material for photovoltaic (PV) infrared detectors [1] both for MWIR (3 to $5 \mu \mathrm{m}$ ) and LWIR (8 to $12 \mu \mathrm{m}$ ) infrared regions.

MCT FPAs now provide performance predominantly limited by readout electronics. The best LWIR and MWIR detectors are almost background limited and it is almost impossible to improve their sensitivities. But readouts in LWIR arrays can utilize now only about $1 \%$ of the available signal at $f / 1$-optics and $T \approx 300 \mathrm{~K}$ background because the unit cell capacitors are filling up in about 20 to $50 \mu$ sec due to background signal influence, while the frame times are about $20 \mathrm{~ms}$. For ensuring high performance FPA characteristics the electrical signals from MCT photodiodes should be extracted by electronics not introducing extra noises at the output of FPA.

There are exist a few parameters for FPA to be characterized: sensitivity, detectivity, noise equivalent temperature difference (NETD) and some others. But initially most of them are based on the measurements of the electrical signals and their noise. The proper measurement of signal-to-noise ratio is the key issue to determine such performance parameters for IR-sensors as NETD and detectivity. Naturally, the efforts are directed to minimize all possible noises. But, as a result, for little value of noise there is a need of corresponding equipment to measure it, at high and low frequencies especially. In the present paper, we attempt to define more exactly the sources of the noise of MCT $n^{+}-p$-junctions in multipixel arrays studied, as they characterize the noise level and its frequency dependence of MCT arrays for LWIR $(\lambda \approx 8-12 \mu \mathrm{m})$ spectral region.

\section{FPAs noise measurements}

Previously [2,3] it was demonstrated that there is a correlation between tunnel currents and low-frequency $(1 / f)$ noise in diffusion $\mathrm{HgCdTe}$ photodiodes. Some correlations between the values of trap assisted tunneling (TAT) current and $1 / f$ noise were also noticed in MCT photodiodes (the smaller is the reverse bias at which TAT begins, the larger is the frequency at which the "knee point" of $1 / f$ noise is situated). But because of this noise was not 


\section{F.F. Sizov et al.: Noise in HgCdTe LWIR arrays}

very pronounced in hybridized arrays studied, it is not considered here. It was shown that native dark current noise of MCT arrays itself is negligible in comparison with other noise sources.

The important issue in studying the FPA noise is the precision of the output signal amplitude measurements. In order to build up the registration system, one should estimate the fluctuations of the input charge at the readout circuits accumulative capacitors and estimate the noise of the output signal, that is caused by these fluctuations. One can estimate fluctuations of the input charge according to Poisson's character of the distribution function of the fluctuations probability of the number of charge particles $N$ which is applicable in this case: $\left(\langle\Delta N\rangle^{2}\right)^{1 / 2}=$ $=N^{1 / 2} ;\left(<\Delta Q>^{2}\right)^{1 / 2}=(e \cdot Q)^{1 / 2}$, where $e$ is the electron charge.

As a rule, the charge-voltage converter with linear transfer function characteristics is used at the FPA output. If the maximum output voltage $U_{0}$ corresponds to maximum input charge $Q_{0}$, then the fluctuation value $\left(<\Delta U^{2}>\right)^{1 / 2}$ or $U_{\text {noise }}$ depending on the output voltage $U$ (corresponds to charge $Q$ ) is given by the expression:

$$
U_{\text {noise }}=\sqrt{<\Delta U\rangle^{2}}=\frac{U_{0}}{Q_{0}} \cdot \sqrt{<\Delta Q>^{2}}=\frac{U_{0}}{Q_{0}} \cdot \sqrt{e \cdot Q}
$$

and as $Q=\frac{Q_{0}}{U_{0}} \cdot U$, than

$$
U_{\text {noise }}=\sqrt{e \cdot \frac{U_{0}}{Q_{0}} \cdot U}
$$

In Fig. 1 the calculated dependencies of noise voltage $U_{\text {noise }}$ on charge $Q$ at three different values of chargevoltage transfer coefficients $\left(U_{0}=4,5\right.$, and $6 \mathrm{~V}$ respectively) are shown. As, for example, one can see from curve 3

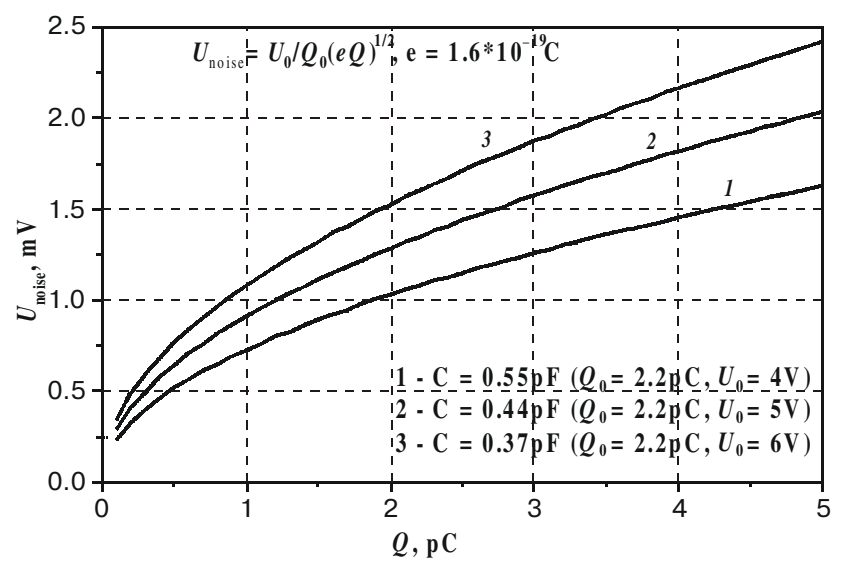

Fig. 1. Fluctuation of $U_{\text {noise }}$ of CCD-register output voltage. $Q_{0}$, $U_{0}, C$ are the maximum input charge, peak output voltage and CCD-register output capacity, respectively $\left(C=Q_{0} / U_{0}\right) . Q$ is the average input charge including dark current and background radiation.
$\left(Q_{0}=2.2 \mathrm{pC}, U_{0}=6 \mathrm{~V}\right)$, for measuring of $U_{\text {noise }}$ with output signal amplitude of $U=6 \mathrm{~V}$, one should carry out the measurements with the precision better than $1.6 \mathrm{mV}$. Therefore, when measuring the signal with amplitude of $5 \mathrm{~V}$, which is produced by the readout circuit, it is necessary to use 12 bit or better 14 bit ADC. A 12-bit ADC was used in the present investigation. 14 bit ADC has to be used for measuring the $1 / f$ noise at low frequencies $(f \leq 10 \mathrm{~Hz})$, which would be almost impossible with 12 bit ADC because of its low frequency "knee point" position measured in diodes without applying readouts. In any case, both for 12 bit and 14 bit ADC it is appropriate to apply the above-described technique to investigate the origin of noise.

In Fig. 2 the different operating modes of the input circuit of CCD silicon readout are shown. Silicon readout devices with input direct injection circuits and CCD multiplexers to be used with $n^{+}-p$-photovoltaic (PV) multipixel arrays were designed and manufactured [4]. The read-out devices with skimming and partitioning functions were manufactured by $n$-channel MOS technology with buried or surface channel CCD register. The analysis of the readouts designed has shown that at well stabilized direct voltages the noise level of the readout itself is negligibly small compared to the background flux current.

The input transistor of direct injection REF0 sets the operating point (reverse bias) of the photodiode. At accumulating capacitor, which is formed by three cells ( $\mathrm{UC1}$, FPS, UC2) the charge $Q=I \times \tau_{\text {acc }}$ is accumulating, where $I$ is the photodiode current, and $\tau_{\text {acc }}$ is the charge accumulating time. The current $I$ consists of the dark current contribution $\mathrm{I}_{\mathrm{d}}$ and photocurrent $I_{\mathrm{ph}}$ at the operating point (see Fig. 3). Selection of the operating point in Fig. 3 is realized with the help of the voltage REF0 at input transistor (e.g., for value of $U_{\mathrm{REF} 0} \approx 0.5 \mathrm{~V}$ corresponds the value of reverse bias at each photodiode $U_{\text {rbias }} \approx 120 \mathrm{mV}$ ) aiming at the maximum of the signal-to-noise ratio, and also taking into account the spread of the threshold voltages $\Delta U_{\text {thr }}$ of the input transistors (for three types of the read-out devices developed the value of $D U_{\mathrm{thr}}$ according to which the readout circuits are culled is equal to $\Delta U_{\text {thr }} \leq \pm 7 \mathrm{mV}$ ). The accumulation time $\tau_{\text {acc }}$ is set by capacity FPS, which is used as a gate, or by transistor F0. The period $T_{\text {read }}$ between the openings of transistor F0 defines also the information taken off from all the diodes of multipixel array. In general case $\tau_{\text {acc }}$ and $T_{\text {read }}$ do not coincide $\left(\tau_{\mathrm{acc}} \leq T_{\text {read }}\right)$. At opening of transistor F0 the input charge $Q$ falls into 16 or 18 bit CCD multiplexer and is converted to voltage at the load resistor outside of the cold zone. In Fig. 4 typical oscillograms of the output signals of one of the FPA analogue outputs are shown. The amplitude of each pulse $U$ characterizes the information on the input charge $Q$ at the accumulation capacitor of one of the pixel registration channels.

In order to investigate the nature of noise sources and decrease it, the noise frequency content for FPA was investigated, and the results obtained are presented in Fig. 5. One can see a few noise harmonics near $4000 \mathrm{~Hz}$ region 


\section{F.F. Sizov et al.: Noise in HgCdTe LWIR arrays}

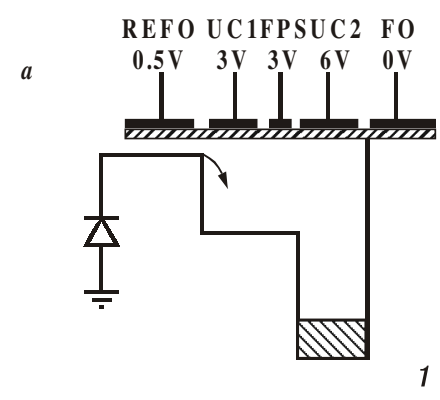

$b$

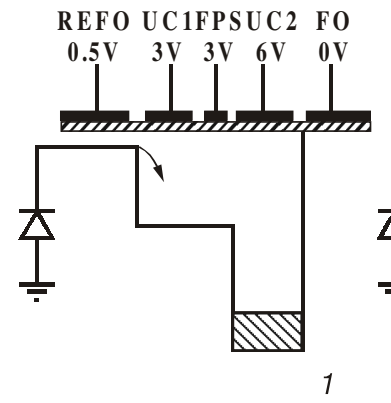

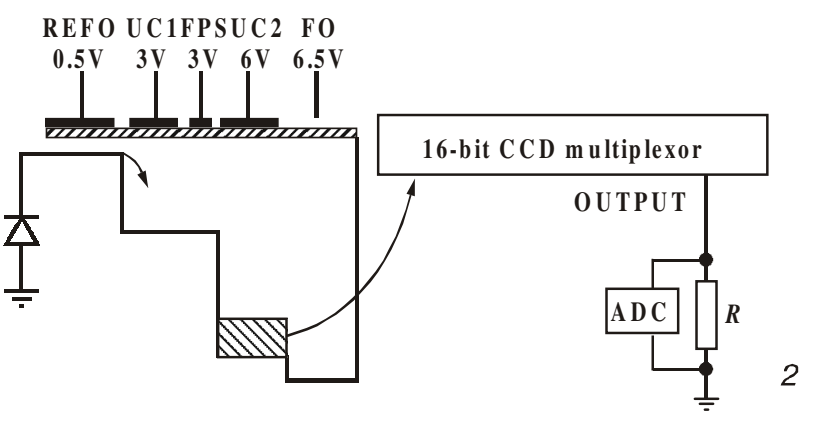

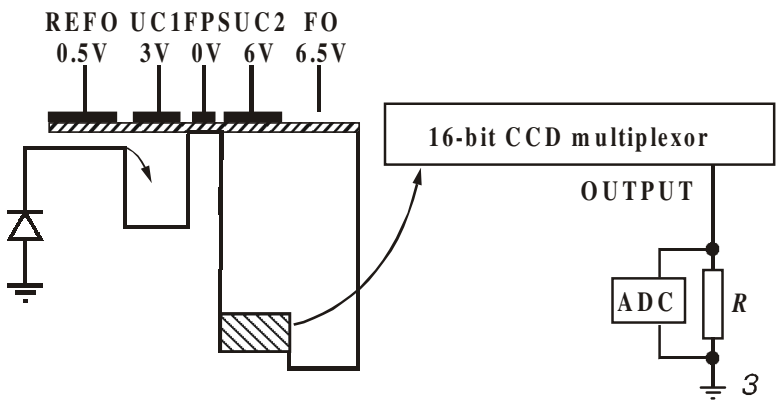

Fig. 2. Different operating modes of the input circuit of CCD silicon readouts.

for low and high level of signal that can be eliminated using double correlating sampling (DCS) procedure.

To analyze the value of the noise as a function of time, the running average and running dispersion were calculated. The results of computation are presented in Fig. 6. Apparently, the value of noise oscillates with time at $100 \mathrm{~Hz}$ frequency that is a $50 \mathrm{~Hz}$ electrical network system harmonic. If supplying all FPA voltages from a direct voltage source the noise voltage can be reduced from 2 $\mathrm{mV}$ to approximately $1.6 \mathrm{mV}$ that is near $20 \%$ improvement.

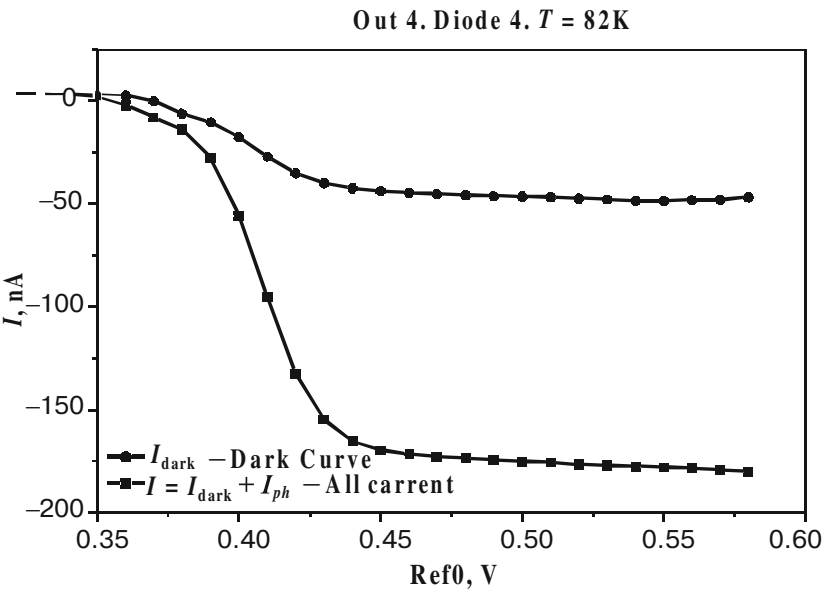

Fig. 3. Dependence of the photodiode dark current (upper curve) and the current under background illumination (lower curve) on the reverse bias (dependent on REF0) for array with $\lambda_{\mathrm{co}}=12.2 \mu \mathrm{m}$.
In Fig. 7a the dependence of the amplitude $U_{\text {sig }}$ of one of the information signals on time is shown, which was measured in the time range $T_{\text {read }}=36 \mu$ s (integration time $\tau_{\text {acc }}=8 \mu$ s that is an acquisition range). Calculation of $U_{\text {noise }}$ during the time range gives the value of $U_{\text {noise }}=$ $=1.99 \mathrm{mV}$. But as one can see from Fig. $7 \mathrm{~b}$ the time ranges, in which $U_{\text {noise }}$ is calculated, and also the moments of time of the beginning of $U_{\text {noise }}$ measuring are not equivalent, which is caused by the presence of the impulse noise disturbances in registration system (in this particular case the most noticeable is the noise distur-

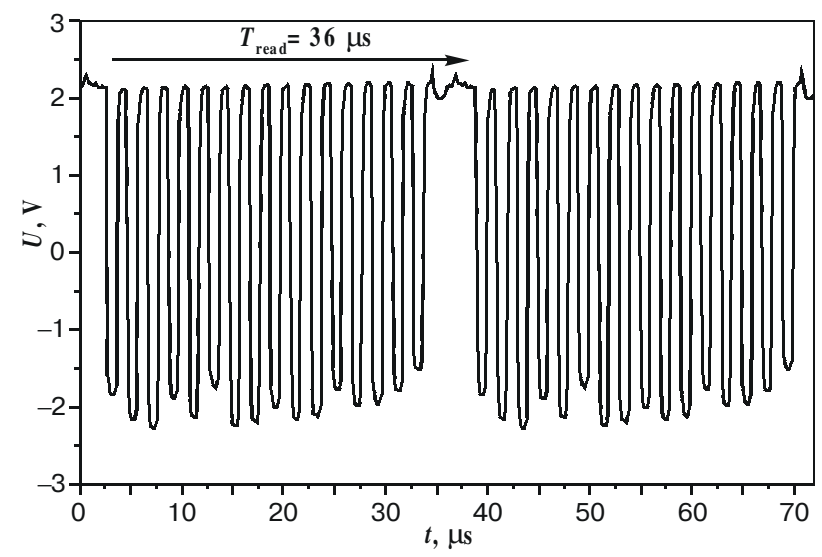

Fig. 4. The oscillogram of sygnals taken from 16 photodiodes of one of CCD-outputs. 


\section{F.F. Sizov et al.: Noise in HgCdTe LWIR arrays}
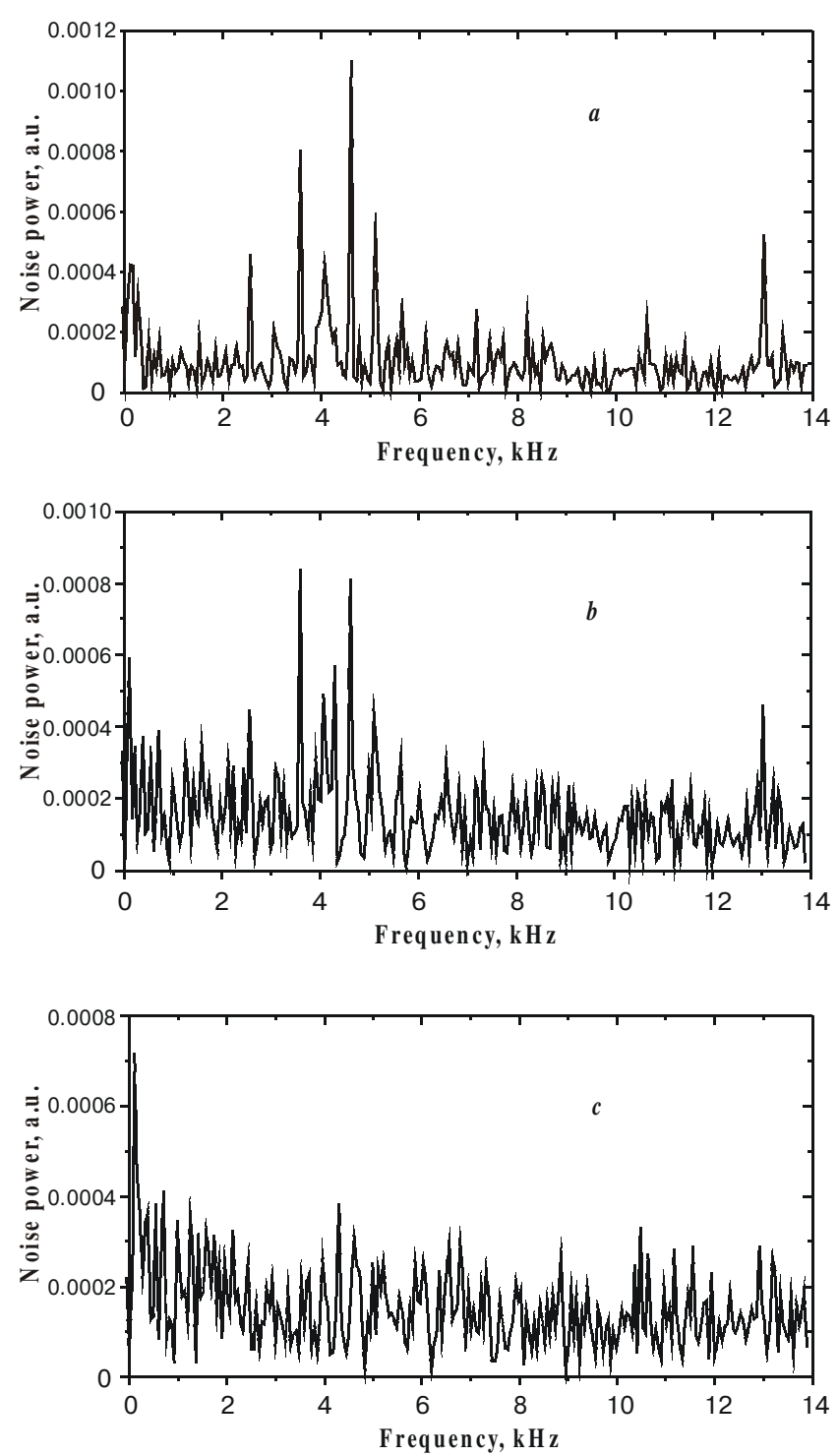

Fig. 5. Spectral noise power for one of FPA channel. At $T=80^{\circ} \mathrm{K}$, The accumulation time is $\tau_{\text {acc }}=8$ ms. $a$ - high level of signal $\left(U_{h 1}\right)$; $b$ - low level of signal $\left(U_{11}\right)$; c) double correlating sampling (DCS) of the signal.

bance at frequencies which are multiplies of $f=100 \mathrm{~Hz}$ ). Thus, if the calculations are taken over 40 points (at the time range $40 \times 36 \mathrm{~ms}=1440 \mathrm{~ms}$ ) then the maximum value of $\left(U_{\text {noise }}\right)_{\max }=2.85 \mathrm{mV}$, and the minimum value of $\left(U_{\text {noise }}\right)_{\min }=1.21 \mathrm{mV}$. The calculating $\left(\mathrm{U}_{\text {noise }}\right)_{\min }$ over 100 points (the time range is equal to 3600 seconds), one gets $\left(U_{\text {noise }}\right)_{\max }=2.36 \mathrm{mV}$ and $\left(U_{\text {noise }}\right)_{\min }=1.45 \mathrm{mV}$.

To analize the noise signals, the fast Fourier transform (FFT) was used. In Fig. 5a, b, c are shown the FFT spectra of $U_{\mathrm{LL}}, U_{\mathrm{HL}}$ and $U_{\mathrm{DCS}}$ signals, respectively. It was possible to eliminate the pulse noises at $f=4 \mathrm{kHz}$ and $f=13 \mathrm{kHz}$ (Figs 7,a and 7,b) using double correlated sampling (DCS): $U_{\text {sig }}=U_{\mathrm{DCS}}=U_{\mathrm{HL}}-U_{\mathrm{LL}}$. Further investigations were carried out with DCS signals.
One of the most important parameters of FPA is the detectivity $D_{\lambda}{ }^{*}$, which is characterized by including the noise band $\sigma_{\Delta f}=U_{\text {noise }} /(\Delta f)^{1 / 2}\left(\mathrm{~V} / \mathrm{Hz}^{1 / 2}\right)$, where $U_{\text {noise }}$ is the mean square of noise in the frequency band $\Delta f$. Conventional expression for $D_{\lambda}{ }^{*}$ looks like

$$
D_{\lambda}^{*}=\frac{U_{\Delta}}{U_{\text {noise }} \cdot H \cdot A}\left(\frac{A}{2 \cdot \tau_{\text {acc }}}\right)^{1 / 2}
$$

where $U_{\Delta}$ is the FPA reaction on the radiation intensity $H\left(\mathrm{~W} / \mathrm{cm}^{2}\right)$, and $A$ is the photosensitive element area. In this expression $\Delta f=\left(2 \tau_{\text {acc }}\right)^{-1}$.

To investigate the real FPA for determination of $\sigma_{\Delta f}=$ $U_{\text {noise }} /(\Delta f)^{1 / 2}$, the FFT procedure was used. To do this, it is necessary that: a) the precision of signal measurements is better than the noise level (at least 12 or 14 bit ADC converter should be used); b) $T_{\text {read }}=\tau_{\text {acc }}$; c) the sampling number $N$ should correspond to the lowest frequency $f_{1}$, which one would like to get in the spectrum.

In Fig. 7a the time dependence and FFT spectrum of the initial signal with its mean value are shown. As it was shown, the calculated value of $U_{\text {noise }}=1.99 \mathrm{mV}$ during the time range $\tau=0-0.02 \mathrm{~s}$, and $\sigma_{\Delta f}=1.99 /(1400)^{1 / 2} \approx$ $\approx 1.68 \cdot 10^{5} \mathrm{~V} /(\mathrm{Hz})^{1 / 2}$. In Fig. $7 \mathrm{~b}$ the same signal and its FFT spectrum (Fig. 7a) are shown, but this time after passing through the digital filter in the range between 8 and $10 \mathrm{kHz}$. In this case $U_{\text {noise }}=0.552 \mathrm{mV}, \sigma_{\Delta f}=$ $=1.23 \times 10^{-5} \mathrm{~V} / \mathrm{Hz}^{1 / 2}$ (the factor 1.37 lower than in the initial case). Using investigation of real FPA such kind
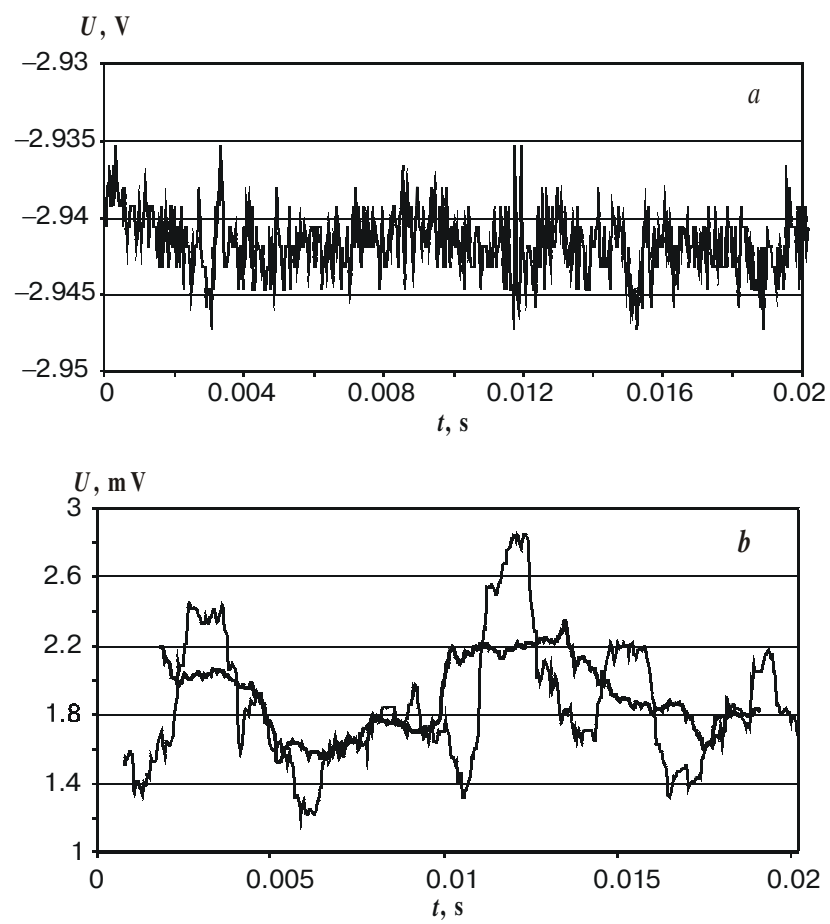

Fig. 6. $a$ - signal for one of FPA channel after DCS, $b$ - voltage of noise calculated on 40 point and 100 point (bold line) respectively. 


\section{F.F. Sizov et al.: Noise in HgCdTe LWIR arrays}
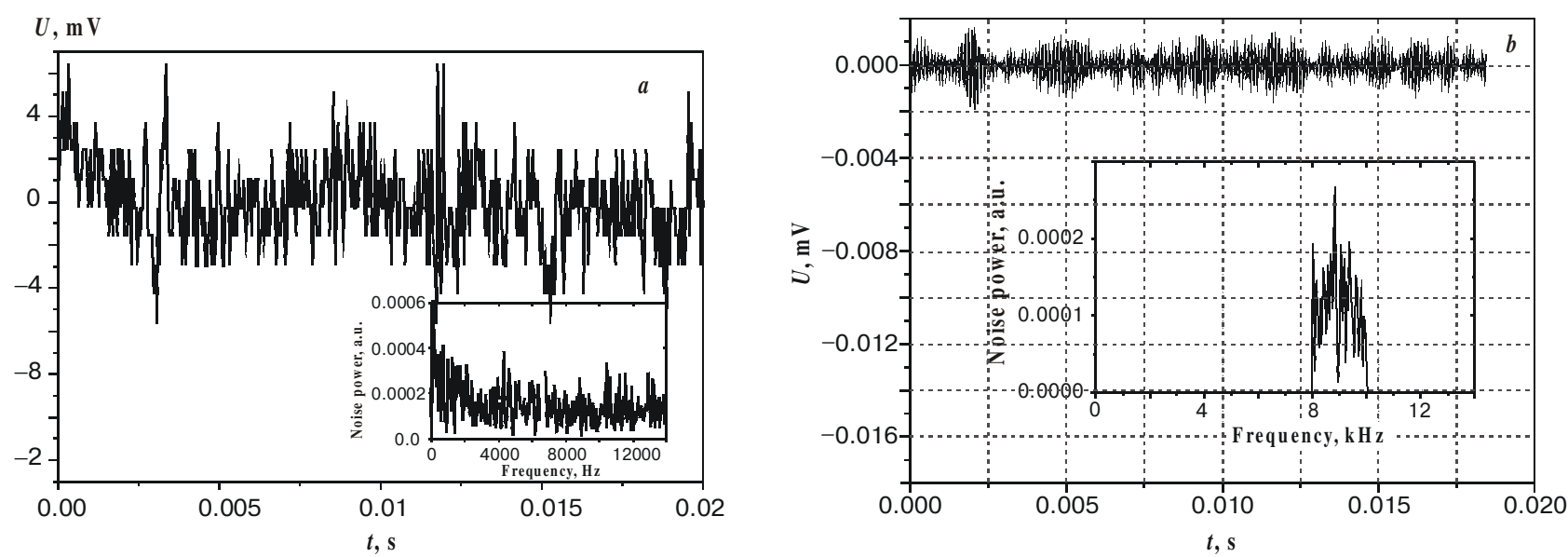

Fig. 7. $a$ - dependence of the amplitude and the FFT spectrum of the initial signal with mean value subtraction, $b$ - the result of application of 8000 to $10000 \mathrm{~Hz}$ band pass filter on DCS of signal is shown in the previous picture.

of analysis is important for the determination of the $1 / f$ component, and also of the pulse noise in FPA.

Applying the procedure described above for MCT hybrid linear arrays of $2 \times 64$ format, the values of detectivity $D_{\lambda}^{*} \cong 7 \cdot 10^{10} \mathrm{~cm} \cdot \mathrm{Hz}^{1 / 2} / \mathrm{W}$ and $N E D T=26 \mathrm{mK}$ $\left(\mathrm{FOV} \approx 45^{\circ}, \lambda_{\mathrm{co}} \approx 10.3 \mu \mathrm{m}, \tau_{\mathrm{acc}}=32 \mu \mathrm{s}\right.$ with partitioning and skimming functions included, $T=82 \mathrm{~K}$ ) were obtained.

\section{Conclusion}

To registrate the FPA output signals, the important point is precision and error of their measurements. In registration system considered here, the method of evaluation of the FPA output signals noise $\left(<\Delta U>^{2}\right)^{1 / 2}$ is used in dependence on output signal level $U$ and FPA charge-voltage transfer coefficient $k=Q_{0} / U_{0}$. The FPAs noise measurements have shown that the values of $\left(\langle\Delta U\rangle^{2}\right)^{1 / 2}$ evaluated are less than those measured directly. The spectral analysis of the output signals using FFT procedure have shown that the reason of it is both an insufficient precision of measuring equipment (mainly because of 12 bit
ADC) and the influence of the impulse noises (mainly harmonic components of the $f=50 \mathrm{~Hz}$ frequency). Silicon readouts designed and manufactured do not introduce additional noise. The procedure proposed of FPA noise analysis permits one to reveal and eliminate the reason of incorrect measuring the noise of the real FPA (impulse noises and the noise of registration system, the precision of keeping of control voltages of silicon readout, the noises of the readout itself, etc.).

\section{References}

1. M.B. Reine, HgCdTe photodiodes for IR detection: A Review // Proc. SPIE, 4288, 266-277 (2001).

2. Y. Nemirovski, D. Rosenfeld, R. Adar, and A. Kornfeld, Tunneling and dark currents in $\mathrm{HgCdTe}$ photodiodes // $J$. Vac. Sci. Technol., A7, 529-534 (1989).

3. F.F. Sizov, S.Ya. Stochanski, V.V. Tetyorkin, S.V. Belyaev, and V.V. Andrievskii, Recombination and carrier transport mechanisms in $\mathrm{Hg}_{1-\mathrm{x}} \mathrm{Cd}_{\mathrm{x}} \mathrm{Te}$ single crystals and $p^{+}-n-$ photodiodes // Ukrainian J. Phys., 44, $752-758$ (1999).

4. F.F. Sizov, Yu.P. Derkach, Yu.G. Kononenko, V.P. Reva, Readout device processing electronics for linear and focal plane arrays // Proc. SPIE, 3436, 942-948 (1999). 